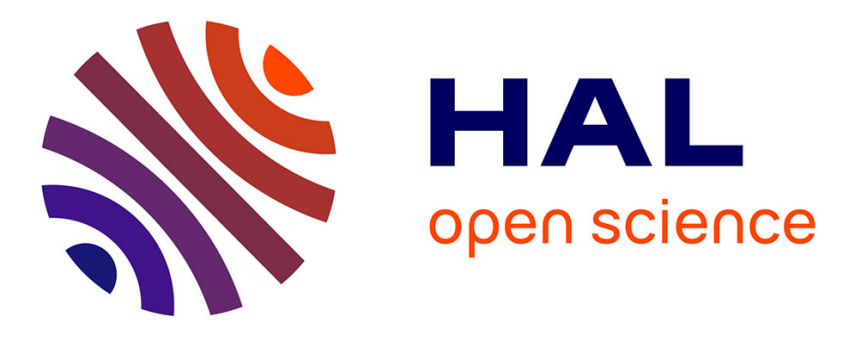

\title{
A dynamic regularized gradient model of the subgrid-scale stress tensor for large-eddy simulation
}

Antoine Vollant, Guillaume Balarac, Christophe Eric Corre

\section{To cite this version:}

Antoine Vollant, Guillaume Balarac, Christophe Eric Corre. A dynamic regularized gradient model of the subgrid-scale stress tensor for large-eddy simulation. Physics of Fluids, 2016, 28 (2), pp.025114. 10.1063/1.4941781 . hal-01339923

\section{HAL Id: hal-01339923 https://hal.science/hal-01339923}

Submitted on 7 Mar 2018

HAL is a multi-disciplinary open access archive for the deposit and dissemination of scientific research documents, whether they are published or not. The documents may come from teaching and research institutions in France or abroad, or from public or private research centers.
L'archive ouverte pluridisciplinaire HAL, est destinée au dépôt et à la diffusion de documents scientifiques de niveau recherche, publiés ou non, émanant des établissements d'enseignement et de recherche français ou étrangers, des laboratoires publics ou privés. 


\section{A dynamic regularized gradient model of the subgrid-scale stress tensor for large-eddy simulation}

A. Vollant, G. Balarac, and C. Corre

Citation: Physics of Fluids 28, 025114 (2016); doi: 10.1063/1.4941781

View online: https://doi.org/10.1063/1.4941781

View Table of Contents: http://aip.scitation.org/toc/phf/28/2

Published by the American Institute of Physics

\section{Articles you may be interested in}

A mixed multiscale model better accounting for the cross term of the subgrid-scale stress and for backscatter

Physics of Fluids 28, 025111 (2016); 10.1063/1.4941773

A dynamic subgrid-scale eddy viscosity model

Physics of Fluids A: Fluid Dynamics 3, 1760 (1991); 10.1063/1.857955

Shifted periodic boundary conditions for simulations of wall-bounded turbulent flows

Physics of Fluids 28, 025112 (2016); 10.1063/1.4941912

A dynamic regularized gradient model of the subgrid-scale scalar flux for large eddy simulations Physics of Fluids 25, 075107 (2013); 10.1063/1.4813812

Physical consistency of subgrid-scale models for large-eddy simulation of incompressible turbulent flows

Physics of Fluids 29, 015105 (2017); 10.1063/1.4974093

Scale-adaptive subgrid-scale modelling for large-eddy simulation of turbulent flows

Physics of Fluids 29, 035101 (2017); 10.1063/1.4977089

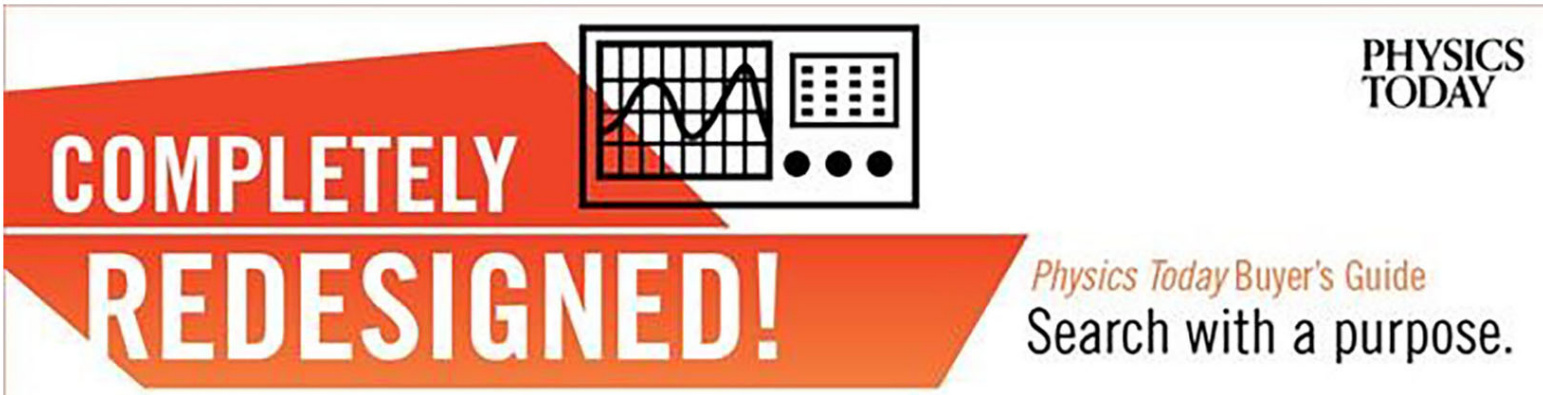




\title{
A dynamic regularized gradient model of the subgrid-scale stress tensor for large-eddy simulation
}

\author{
A. Vollant, ${ }^{1,2}$ G. Balarac, ${ }^{1}$ and C. Corre ${ }^{3}$ \\ ${ }^{1}$ University Grenoble Alpes, CNRS, LEGI UMR 5519, Grenoble F-38041, France \\ ${ }^{2}$ OptiFluides, Villeurbanne F-69603, France \\ ${ }^{3}$ Ecole Centrale de Lyon, LMFA UMR 5509, Ecully F-69134, France
}

(Received 9 September 2015; accepted 31 January 2016; published online 26 February 2016)

\begin{abstract}
Large-eddy simulation (LES) solves only the large scales part of turbulent flows by using a scales separation based on a filtering operation. The solution of the filtered Navier-Stokes equations requires then to model the subgrid-scale (SGS) stress tensor to take into account the effect of scales smaller than the filter size. In this work, a new model is proposed for the SGS stress model. The model formulation is based on a regularization procedure of the gradient model to correct its unstable behavior. The model is developed based on a priori tests to improve the accuracy of the modeling for both structural and functional performances, i.e., the model ability to locally approximate the SGS unknown term and to reproduce enough global SGS dissipation, respectively. LES is then performed for a posteriori validation. This work is an extension to the SGS stress tensor of the regularization procedure proposed by Balarac et al. ["A dynamic regularized gradient model of the subgrid-scale scalar flux for large eddy simulations," Phys. Fluids 25(7), 075107 (2013)] to model the SGS scalar flux. A set of dynamic regularized gradient (DRG) models is thus made available for both the momentum and the scalar equations. The second objective of this work is to compare this new set of DRG models with direct numerical simulations (DNS), filtered DNS in the case of classic flows simulated with a pseudo-spectral solver and with the standard set of models based on the dynamic Smagorinsky model. Various flow configurations are considered: decaying homogeneous isotropic turbulence, turbulent plane jet, and turbulent channel flows. These tests demonstrate the stable behavior provided by the regularization procedure, along with substantial improvement for velocity and scalar statistics predictions. (C) 2016 AIP Publishing LLC. [http://dx.doi.org/10.1063/1.4941781]
\end{abstract}

\section{INTRODUCTION}

Due to the large range of motion scales in turbulent flows, the direct numerical simulation (DNS) of realistic applications is not yet feasible because of its excessive computational cost. To overcome this limitation, the large-eddy simulation (LES) technique proposes to explicitly solve only the large scales of the flow and to model the impact of the smallest scales on the large scales. The separation between resolved large scales and modeled small scales is performed by a filtering operation. The filtering operation applied to the flow equations leads to subgrid-scale (SGS) terms which have to be modeled. The incompressible filtered Navier-Stokes equation writes

$$
\frac{\partial \bar{u}_{i}}{\partial t}+\frac{\partial \bar{u}_{i} \bar{u}_{j}}{\partial x_{j}}=-\frac{1}{\rho} \frac{\partial \bar{p}}{\partial x_{i}}+v \frac{\partial^{2} \bar{u}_{i}}{\partial x_{j}^{2}}-\frac{\partial \tau_{i j}}{\partial x_{j}},
$$

with $\tau_{i j}=\overline{u_{i} u_{j}}-\bar{u}_{i} \bar{u}_{j}$ the SGS stress tensor to model in order to perform LES. In this equation, $\bar{u}_{i}$ is the component of the filtered velocity in the $i$ th direction, $\bar{p}$ is the filtered pressure, $\rho$ is the constant density, and $v$ is the constant kinematic viscosity. Various models have been proposed to close the filtered Navier-Stokes equations for incompressible flows. ${ }^{1-3}$

Two major strategies are usually followed to develop SGS models: ${ }^{3}$ functional and structural strategies. The functional modeling strategy considers the action of the subgrid terms over the 
energy transfer between resolved and modeled scales and not the unknown term itself. It can introduce a dissipative term, for example, that has a similar effect but not necessarily the same spatial structure. Conversely, the structural modeling strategy consists in using the best local approximation of the unknown SGS term by constructing it from the known structure of small-scales.

A typical functional model consists in defining an eddy viscosity to model the SGS stress tensor as $\tau_{i j}-1 / 3 \tau_{k k} \delta_{i j}=-2 v_{T} \bar{S}_{i j}$, with $\bar{S}_{i j}=1 / 2\left(\partial \bar{u}_{i} / \partial x_{j}+\partial \bar{u}_{j} / \partial x_{j}\right)$ the filtered strain rate tensor. Various formulations for the eddy viscosity have been proposed. ${ }^{4-6}$ The Smagorinsky model,

$$
v_{T}=C \bar{\Delta}^{2}|\bar{S}|,
$$

with $|\bar{S}|=\sqrt{2 \bar{S}_{i j} \bar{S}_{i j}}, \bar{\Delta}$ the filter size and $C$ the model coefficient, is certainly the most commonly used eddy-viscosity model. Due to its computational stability and the simplicity of its formulation, this model is a valuable tool for engineering applications. ${ }^{7}$ However, this model is found to be too dissipative in shear regions because of an excessive eddy-viscosity. ${ }^{8}$ Various corrections ${ }^{9,10}$ have been proposed to limit the SGS dissipation in these regions. The most commonly used approach is to determine dynamically the model coefficient using the Germano identity. ${ }^{9,11}$ Note that the dynamic Smagorinsky (DS) model has also been extended to model the SGS scalar flux with a dynamic eddy Schmidt number. ${ }^{12}$ However, even though a correct dissipation level is modeled, the DS model is generally known to exhibit weak local correlations with the SGS terms. ${ }^{13}$ Indeed, like other eddy viscosity models, the DS model assumes an alignment between the eigenvectors of the SGS stress tensor and the filtered strain rate tensor $\bar{S}_{i j}$, which is not observed in reality. ${ }^{14}$ To correct this behavior, various approaches have been proposed based on the definition of a velocity increment, ${ }^{15}$ a scale-similarity assumption ${ }^{16}$ or Taylor series expansions of the filtering operation. ${ }^{17}$

Models defined from Taylor series expansions of the filtering operation are typical structural models, often called gradient $(\mathrm{G})$ models.$^{13}$ In the case of the SGS stress tensor, this model writes as

$$
\tau_{i j}^{\mathrm{G}}=\frac{\bar{\Delta}^{2}}{12} \frac{\partial \bar{u}_{i}}{\partial x_{k}} \frac{\partial \bar{u}_{j}}{\partial x_{k}} \text {. }
$$

This type of model is known to provide a good local approximation of the unknown term with a high correlation between the unknown term and the model in a priori tests. However, this type of model is also known to be unstable due to an incorrect prediction of the dissipation. ${ }^{18}$ Indeed, this model leads to excessive back-scatter, i.e., energy transfer from modeled to resolved scales. ${ }^{19,20}$ Thus, various modifications have been proposed to stabilize the gradient model. These modifications can rely on using a "clipping" 1,22 or on combining this model with an eddy viscosity model as in mixed models. ${ }^{13,21,23}$

More recently, a novel regularization procedure has been proposed when the gradient model is used to model the SGS scalar flux in the context of LES of turbulent mixing. ${ }^{24}$ The first objective of the present work is to extend the regularization strategy for the SGS stress tensor, $\tau_{i j}$. The regularization is based on a decomposition of the filtered velocity gradients appearing in gradient model (3). This allows to identify the terms responsible for unstable behavior. A stable model is then proposed by removing these terms. This new model can be defined as both structural and functional. Indeed, on one hand, the model starts from Taylor series expansions of the filtering operation similarly to standard structural gradient models but on the other hand, the model is also developed to control the predicted SGS dissipation similarly to standard functional models. The modeling strategy is first assessed from a priori tests (i.e., in comparison with SGS terms evaluated from a DNS database).

With this model for the SGS stress tensor and the regularized model for the SGS scalar flux ${ }^{24}$ a new set of dynamic regularized gradient models for velocity and scalar is now available. The second objective of this paper is then to test a posteriori this new set of models, by performing LES in various flow configurations. It is thus demonstrated that the proposed regularization leads to stable computations, with a substantial prediction improvement for various velocity and scalar statistics in comparison with the set of standard models based on the dynamic Smagorinsky model. ${ }^{9,11,12}$ 


\section{MODEL FORMULATION}

\section{A. Decomposition of the SGS dissipation predicted by the gradient model}

In the context of SGS stress tensor modeling, the functional performance can be defined as the model ability to reproduce the global grid-scale/subgrid-scale (GS/SGS) transfer of kinetic energy. This transfer is controlled by the SGS dissipation, ${ }^{25,26}-\tau_{i j} \bar{S}_{i j}$. For a LES simulation to be accurate, a model must correctly reproduce the average SGS dissipation. ${ }^{27}$ A positive value of the GS/SGS dissipation leads to direct transfer from the large resolved scales to the small unresolved scales (forward-scatter), while a negative value characterizes an inverse transfer (back-scatter). The SGS dissipation is usually positive but with local negative values. ${ }^{25}$ The gradient model, Eq. (3), is known to over-predict the back-scatter effect, leading to an under-estimation of the SGS dissipation and, consequently, leading to an unstable simulation with an accumulation of kinetic energy at the smallest resolved scales.

To better understand this behavior, the SGS dissipation predicted by the gradient model can be re-written using a splitting of the filtered velocity gradients in terms of filtered strain rate tensor and filtered rotation tensor,

$$
\begin{aligned}
-\tau_{i j}^{\mathrm{G}} \bar{S}_{i j} & =-\frac{\bar{\Delta}^{2}}{12} \frac{\partial \bar{u}_{i}}{\partial x_{k}} \frac{\partial \bar{u}_{j}}{\partial x_{k}} \bar{S}_{i j} \\
& =-\frac{\bar{\Delta}^{2}}{12}\left(\bar{S}_{i k}+\bar{\Omega}_{i k}\right)\left(\bar{S}_{j k}+\bar{\Omega}_{j k}\right) \bar{S}_{i j} \\
& =-\frac{\bar{\Delta}^{2}}{12}\left(\bar{S}_{i k} \bar{S}_{j k} \bar{S}_{i j}+\left(\bar{S}_{i k} \bar{\Omega}_{j k}+\bar{\Omega}_{i k} \bar{S}_{j k}\right) \bar{S}_{i j}+\bar{\Omega}_{i k} \bar{\Omega}_{j k} \bar{S}_{i j}\right) .
\end{aligned}
$$

The filtered rotation tensor, $\bar{\Omega}_{i j}$, being anti-symmetric and the filtered strain rate tensor, $\bar{S}_{i j}$, being symmetric, it can be shown that $\bar{S}_{i k} \bar{\Omega}_{j k} \bar{S}_{i j}=\bar{\Omega}_{i k} \bar{S}_{j k} \bar{S}_{i j}=0$.

The first term of the RHS in (4) is only defined from the strain rate tensor $\bar{S}_{i j}$. The filtered strain rate tensor being real and symmetric, it can be further decomposed as

$$
\bar{S}_{i j}=\sum_{k=1}^{3} \lambda^{(k)} e_{i}^{(k)} e_{j}^{(k)}
$$

with $\lambda^{(k)}$ and $e_{i}^{(k)}$, respectively, the (real) eigenvalues and the components of the unitary eigenvectors of the filtered strain rate tensor. The first RHS term of the SGS dissipation, Eq. (4), can thus be expended as

$$
\begin{aligned}
\bar{S}_{i k} \bar{S}_{j k} \bar{S}_{i j} & =\left(\sum_{l=1}^{3} \lambda^{(l)} e_{i}^{(l)} e_{k}^{(l)}\right)\left(\sum_{m=1}^{3} \lambda^{(m)} e_{j}^{(m)} e_{k}^{(m)}\right)\left(\sum_{n=1}^{3} \lambda^{(n)} e_{i}^{(n)} e_{j}^{(n)}\right) \\
& =\sum_{l=1}^{3} \sum_{m=1}^{3} \sum_{n=1}^{3} \lambda^{(l)} \lambda^{(m)} \lambda^{(n)} e_{i}^{(l)} e_{i}^{(n)} e_{k}^{(l)} e_{k}^{(m)} e_{j}^{(m)} e_{j}^{(n)}
\end{aligned}
$$

The orthonormality of the eigenvectors can be expressed as $e_{i}^{(l)} e_{i}^{(n)}=\delta_{n l}$, with $\delta_{n l}$ the Kronecker symbol equal to 1 only if $n=l$ and zero otherwise. This leads to

$$
\bar{S}_{i k} \bar{S}_{j k} \bar{S}_{i j}=\sum_{l=1}^{3} \sum_{m=1}^{3} \sum_{n=1}^{3} \lambda^{(l)} \lambda^{(m)} \lambda^{(n)} \delta_{n l} \delta_{l m} \delta_{m n}=\sum_{l=1}^{3} \lambda^{(l)}\left(\lambda^{(l)}\right)^{2} .
$$

Consequently, the sign of $\bar{S}_{i k} \bar{S}_{j k} \bar{S}_{i j}$ is controlled by the eigenvalues $\lambda^{(l)}$. By defining

$$
\bar{S}_{i j}^{\oplus}=\sum_{k=1}^{3} \max \left(\lambda^{(k)}, 0\right) e_{i}^{(k)} e_{j}^{(k)}
$$

and 


$$
\bar{S}_{i j}^{\ominus}=\sum_{k=1}^{3} \min \left(\lambda^{(k)}, 0\right) e_{i}^{(k)} e_{j}^{(k)},
$$

the SGS dissipation predicted by the gradient model is expressed as

$$
-\tau_{i j}^{\mathrm{G}} \bar{S}_{i j}=\frac{\bar{\Delta}^{2}}{12}(\underbrace{-\bar{S}_{i k}^{\oplus} \bar{S}_{j k} \bar{S}_{i j}}_{<0} \underbrace{-\bar{S}_{i k}^{\ominus} \bar{S}_{j k} \bar{S}_{i j}}_{>0} \underbrace{-\left(\bar{S}_{i k} \bar{\Omega}_{j k}+\bar{\Omega}_{i k} \bar{S}_{j k}\right) \bar{S}_{i j}}_{=0}-\bar{\Omega}_{i k} \bar{\Omega}_{j k} \bar{S}_{i j}) .
$$

The term $-\bar{S}_{i k}^{\oplus} \bar{S}_{j k} \bar{S}_{i j}$ is negative, meaning that this term leads to inverse transfer (back-scatter), whereas the term $-\bar{S}_{i k}^{\ominus} \bar{S}_{j k} \bar{S}_{i j}$ is positive and leads to direct transfer. Moreover, as already explained, the term $-\left(\bar{S}_{i k} \bar{\Omega}_{j k}+\bar{\Omega}_{i k} \bar{S}_{j k}\right) \bar{S}_{i j}$ leads to no transfer. Finally, though an analytical development does not allow to assess the sign of the term $\bar{\Omega}_{i k} \bar{\Omega}_{j k} \bar{S}_{i j}$, numerical tests show that this term can be negative and thus lead to inverse transfer.

As already proposed for the SGS scalar flux,${ }^{24}$ a regularization of the gradient model can be achieved by removing all the part of the model leading to inverse transfer in order to avoid unstable behavior. In the model formulation, this means keeping only terms leading to positive or zero SGS dissipation. From Equation (10), two models can be proposed. The first one is obtained by keeping only the term exclusively allowing direct energy transfer. This leads to the first regularized gradient (denoted RG1) model, which is written as

$$
\tau_{i j}^{\mathrm{RG} 1}=\frac{\bar{\Delta}^{2}}{12} \bar{S}_{i k}^{\ominus} \bar{S}_{j k}
$$

The second one is obtained by also keeping the term which leads to no transfer, because it might contribute to the structural performance of the model. The second regularized gradient (denoted RG2) model is written as

$$
\tau_{i j}^{\mathrm{RG} 2}=\frac{\bar{\Delta}^{2}}{12}\left(\bar{S}_{i k}^{\ominus} \bar{S}_{j k}+\bar{S}_{i k} \bar{\Omega}_{j k}+\bar{\Omega}_{i k} \bar{S}_{j k}\right) .
$$

Both these model formulations lead to the same SGS dissipation, i.e., RG1 and RG2 models have the same functional performance.

By construction, the proposed regularization neglects the back-scatter effect. This is a limitation of the current approach, also present in other models where coefficient "clipping" is used (see Refs. 21 and 22, for example). More complex models have been proposed to take into account the back-scatter effect. ${ }^{28,29}$ It will be demonstrated in the following tests that the proposed regularization strategy yields a large prediction improvement with respect to the gradient model, while remaining simple in its formulation. Future works will be devoted to model back-scatter effect, by including in a controlled way into the model terms leading to negative SGS dissipation.

In Subsection II B, the structural performance of the models is first assessed in order to differentiate RG1 and RG2. A priori tests will be also conducted to compare the performance of the proposed models with the DS and the gradient models.

\section{B. A priori tests: Final model formulation}

To further analyze the model formulation proposed, a priori tests are performed based on data extracted from DNS of a forced homogeneous isotropic turbulence. The DNS database is generated from a standard pseudo-spectral code and the simulation domain is discretized using $512^{3}$ grid points on a domain of length $2 \pi$. A statistically steady flow is achieved by using a forcing term. ${ }^{30}$ The Reynolds number based on the Taylor microscale is around 160 at the stationary state. The code and the flow configuration are similar to those used in previous works. ${ }^{23,24,31}$ The DNS data are filtered in space to emulate LES quantities by using a spectral cut-off filter. Several filter sizes have been chosen such that $2 \leq \bar{\Delta} / \Delta x \leq 32$ where $\bar{\Delta}$ is the filter width and $\Delta x$ is the DNS mesh size.

The a priori tests are first performed to determine the best formulation between RG1 and RG2 models, Eqs. (11) and (12), respectively. These models can be differentiated only by their structural performance. From Sagaut, ${ }^{3}$ the structural performance is defined as the model ability to locally 
describe the SGS unknown term appearing in the equation to solve. The divergence of the SGS stress tensor, $\partial \tau_{i j} / \partial x_{j}$ is then considered. As already proposed in previous works ${ }^{23,24,32,33}$ dealing with the development of SGS models, the structural performance is evaluated in the framework of optimal estimation theory ${ }^{34}$ by using the optimal estimator concept introduced by Moreau et al. ${ }^{35}$ in the LES context. Defining the quadratic error between the exact SGS term and the model as the relevant error to consider in LES, ${ }^{36}$ a minimal error can be defined, called the irreducible error. The irreducible error depends only on the set of variables used to write the model. The concept of optimal estimator forecasts that any model built on this set of variables will yield a quadratic error higher than the irreducible error. The irreducible error is computed from the optimal estimator defined as the conditional expectation of the exact SGS term with the chosen set of variables. The optimal estimator theory provides useful informations on the SGS models used in LES. In particular, the most suitable set of variables to model the SGS term can be determined by comparing the corresponding irreducible error. Also, the difference between the quadratic error of a model built on a given set of variables and the irreducible error computed with this same set of variables allows to assess to what extent an existing model can be improved. To determine the most suitable variables to model the SGS stress tensor, the irreducible errors of the RG1 and RG2 models are first computed on the divergence of the SGS stress tensor, $\partial \tau_{i j} / \partial x_{j}$. Due to the flow configuration, the errors are computed using a spatial averaging. Because the divergence of the SGS stress tensor is a vector, the errors are computed based on the square of the Euclidean norm. Moreover, the errors are normalized by the square of the Euclidean norm of the statistical variance of the SGS stress tensor exact divergence. Fig. 1 shows the evolution of the normalized irreducible error with the filter size. The proposed regularized models are also compared with the DS and the gradient models. For the DS model, the set of variables used to compute the irreducible error is $\left\{\frac{\partial}{\partial x_{j}}\left(|\bar{S}| \bar{S}_{i j}\right)\right\}$, whereas the gradient model set of variables is $\left\{\frac{\partial}{\partial x_{j}}\left(\frac{\partial \bar{u}_{i}}{\partial x_{k}} \frac{\partial \bar{u}_{j}}{\partial x_{k}}\right)\right\}$. For the proposed regularized models, the set of variables used is $\left\{\frac{\partial}{\partial x_{j}}\left(\bar{S}_{i k}^{\ominus} \bar{S}_{j k}\right)\right\}$ for RG1 model and $\left\{\frac{\partial}{\partial x_{j}}\left(\bar{S}_{i k}^{\ominus} \bar{S}_{j k}+\bar{S}_{i k} \bar{\Omega}_{j k}+\bar{\Omega}_{i k} \bar{S}_{j k}\right)\right\}$ for RG2 model, respectively. As expected the gradient model irreducible error is smaller than the DS model irreducible error. This is because the gradient model is a structural type model, whereas the DS model is a functional type model. It is thus expected the gradient model will yield a better structural performance which is indeed observed. Because its irreducible error is high, an improvement of the structural performance of the DS model cannot be expected without adding new quantities in

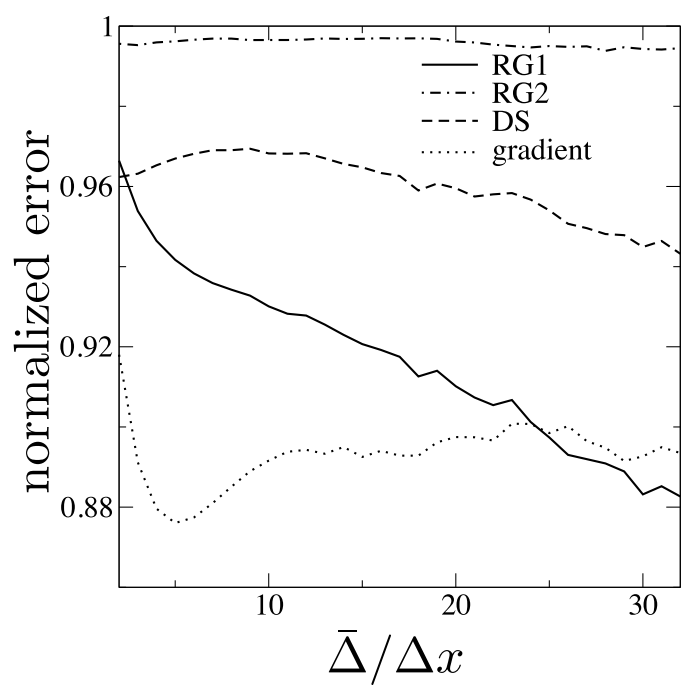

(a)

FIG. 1. Normalized irreducible errors as a function of the filter width for both formulations of the regularization of the gradient model, RG1 and RG2, Eqs. (11) and (12), respectively. The results for DS and gradient models are also shown. 
its set of variables. The performances of the RG1 and RG2 regularized gradient models are very different from one another. The RG1 model yields a good structural performance, much better than that of the RG2 model. Indeed, the RG2 model irreducible error is even higher than DS model error, showing that this model proposal does not provide an improvement in comparison with a classic eddy viscosity model. Conversely, the irreducible error of the RG1 model is significantly smaller than the DS model error. Model formulation (11) should thus lead to an improvement of the structural performance in comparison with the standard DS model. Moreover, the RG1 model formulation should also lead to an improvement of the functional performance in comparison with the gradient model because the GS/SGS energy transfer is better controlled. Note that a decrease of the RG2 model irreducible error could be obtained by using $\left\{\frac{\partial}{\partial x_{j}}\left(\bar{S}_{i k}^{\ominus} \bar{S}_{j k}\right) ; \frac{\partial}{\partial x_{j}}\left(\bar{S}_{i k} \bar{\Omega}_{j k}+\bar{\Omega}_{i k} \bar{S}_{j k}\right)\right\}$ as set of variables. But this choice implies to further decompose the model in two terms with two distinct coefficients to evaluate, leading to a more complex model that will not be considered in the present work.

The proposed form of the regularized model is finally,

$$
\tau_{i j}^{\mathrm{DRG}}=C \bar{\Delta}^{2} \bar{S}_{i k}^{\ominus} \bar{S}_{j k},
$$

with $C$ a coefficient. This coefficient is dynamically defined using a dynamic procedure based on Taylor series expansions, as already proposed to model the SGS scalar flux ${ }^{24}$ and the SGS scalar variance $^{32}$ or to improve the accuracy of a mixed model..$^{23}$ The dynamic procedure uses a test filter, denoted $\hat{\bullet}$, defined similarly to the SGS filter, with a filter size such as $\hat{\Delta}=2 \bar{\Delta}$. Applying the Taylor series expansions of the test filter to the Leonard term, $L_{i j}={\widehat{\bar{u}}, \bar{u}_{j}}_{-}-\hat{\bar{u}}_{i} \hat{\bar{u}}_{j}$, and using the proposed regularization yields

$$
L_{i j}=C \hat{\Delta}^{2} \hat{\bar{S}}_{i k}^{\ominus} \hat{\bar{S}}_{j k}
$$

Assuming the same coefficient value for Equations (13) and (14), this relation can be used to compute the model coefficient. The model coefficient is then evaluated as

$$
C=\frac{\left\langle L_{i j} \hat{\bar{S}}_{i j}\right\rangle}{\left\langle N_{i j} \hat{\bar{S}}_{i j}\right\rangle},
$$

where $N_{i j}=\hat{\Delta}^{2} \hat{\bar{S}}_{i k}^{\ominus} \hat{\bar{S}}_{j k}$ and where the brackets indicate a statistical average over a suitable ensemble to avoid local large values of the coefficient. For this a priori test based on homogeneous isotropic turbulence, the averaging is performed over the homogeneous direction. Note that in our numerical tests, this evaluation of the model coefficient leads to a better prediction of the SGS dissipation in comparison with the filtered DNS result than an evaluation based on the classic least-squares approximation according to Lilly's method. ${ }^{11}$ Equations (13) and (15) define the proposed model, referred from now on as DRG model for Dynamic Regularized Gradient model.

The final model formulation is now compared with the DS and gradient models in a priori tests to better understand the advantage derived from using the regularized formulation. These tests use the DNS database already described and allow to measure the functional and structural performances. The functional performance, i.e., the model ability to predict the GS/SGS transfer, is first studied; this performance is controlled by the SGS dissipation predicted by each model. Figure 2(a) shows the mean SGS dissipation, $-\left\langle\tau_{i j} \bar{S}_{i j}\right\rangle$, as a function of the filter width for the various models and for the filtered DNS. As expected, the mean SGS dissipation is positive showing that the energy transfers are mainly from the large (resolved) scales to the small ones. The gradient model strongly under-predicts the SGS dissipation in comparison with the DNS results. This explains the unstable behavior of this model: ${ }^{20}$ it does not provide enough dissipation by over-estimating back-scatter. On the other hand, the DS model is slightly too dissipative with an over-prediction of the SGS dissipation. Finally, the DRG model yields a SGS dissipation close to the DNS results, which should lead to stable LES. The structural performance is studied next; it is based on the optimal estimation analysis to evaluate the ability of the DRG model to accurately predict the divergence of the SGS stress tensor. The normalized quadratic errors are computed for the models and compared with the normalized irreducible error. Figure $2(\mathrm{~b})$ shows the errors as a function 


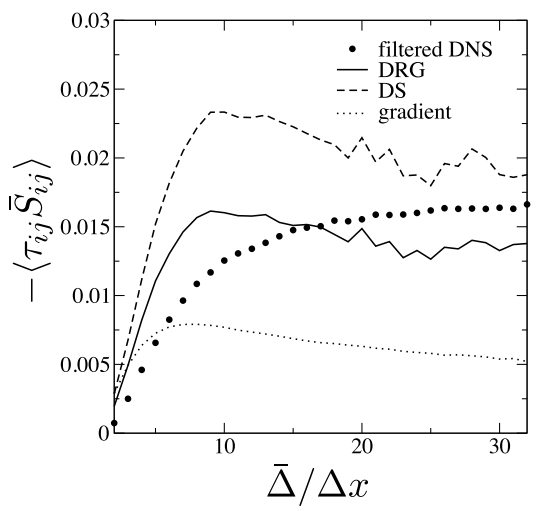

(a)

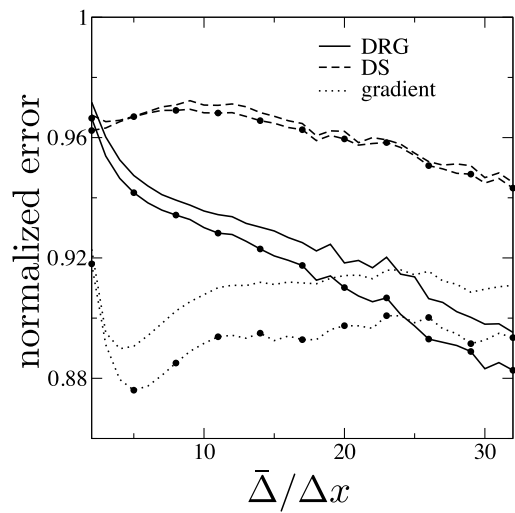

(b)

FIG. 2. A priori test of the DRG model: (a) Mean SGS dissipation as a function of the filter width for the proposed DRG model. The results for DS and gradient models are shown for comparison. The symbols show the SGS dissipation given by the filtered DNS data. (b) Normalized quadratic and irreducible (symbols) errors as a function of the filter width for the proposed DRG model. The results for DS and gradient models are also shown.

of the filter width. As expected, the gradient model has the smallest error since it is designed to optimize the structural performance. The quadratic and irreducible errors of the DS model are very close, showing that the structural performance of the model cannot be further improved and that the dynamic procedure is efficient. However, this model also displays the highest errors. The DRG model allows an improvement of the structural performance in comparison with the DS model, with a significant decrease of the errors. For large filter size, the quadratic error of the DRG model is close to the quadratic error of the gradient model. Finally, the DRG model appears as a good trade-off in terms of functional and structural performance. Indeed, this model allows to improve the functional performance in comparison with the gradient model, while it also leads to a significant improvement of the structural performance in comparison with the DS model.

\section{A POSTERIORI (LES) TESTS}

The newly proposed DRG model based on a regularization of the gradient model and defined by Eqs. (13) and (15) is retained to compute the SGS stress tensor in the filtered Navier-Stokes equation. It is applied to perform LES of some reference turbulent flows in order to provide an $a$ posteriori confirmation of the good structural and functional performance established on the previous a priori tests. This new model is associated with the SGS scalar flux modeled by the dynamic regularized gradient model ${ }^{24}$ to propose a new set of models for both velocity and scalar. In the following test cases, a scalar $Z$ is thus considered to assess the performance of the proposed combination of DRG models. This new set of models will be compared with the set of DS models based on an eddy viscosity and an eddy diffusivity assumptions. In the DS models set, the eddy viscosity is defined by Equation (2) with a dynamic coefficient, ${ }^{9,11}$ and the eddy diffusivity is defined based on a dynamic eddy Schmidt number. ${ }^{12}$

\section{A. Decaying homogeneous isotropic turbulence}

First, decaying homogeneous isotropic turbulence is computed using a spectral numerical method. The flow is first established by DNS, using forcing similarly to the case used in the $a$ priori tests, with $R_{\lambda}=160$. A spectral interpolation is then performed to define the initial condition on the LES mesh. Moreover, the turbulent mixing of a scalar field is considered to assess the SGS scalar flux models. The molecular Schmidt number is fixed to 0.7 and the scalar field is initialized between 0 and 1 according to the procedure proposed by Eswaran and Pope. ${ }^{37}$ The scalar is thus initialized as large spots with a size of the order of half the computational box size. For LES, the simulation domain is discretized with $32^{3}$ grid points. The LES results will be compared with the DNS results 


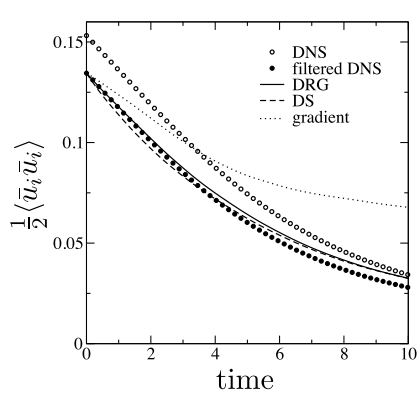

(a)

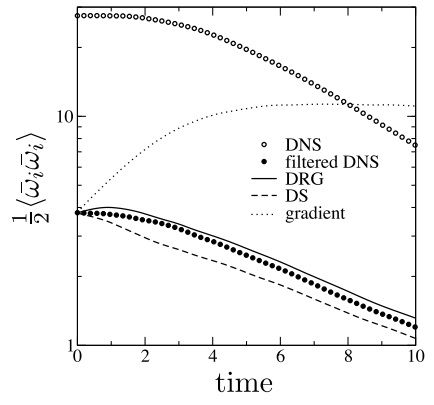

(b)

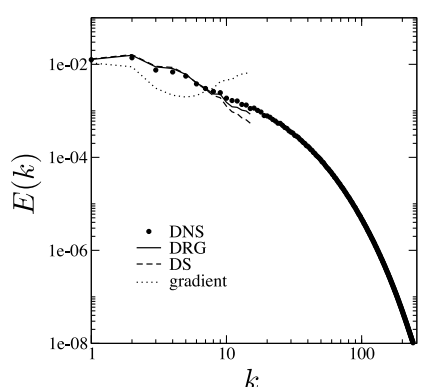

(c)

FIG. 3. Comparison of decaying homogeneous isotropic turbulence statistics: evolution of the resolved kinetic energy (a) and enstrophy (b) with time, and kinetic energy spectrum at $t=5$ (c). DNS (symbol), DRG model (solid line), DS model (dashed line), and gradient model (dotted line).

obtained on a $512^{3}$ grid and starting with the same initial condition. In this test case, the set of gradient models for both scalar and momentum equations is also applied for comparison.

Figure 3 displays the velocity statistics. The respective decay with time of the LES resolved kinetic energy and resolved enstrophy, $1 / 2\left\langle\bar{\omega}_{i} \bar{\omega}_{i}\right\rangle$ with $\omega_{i}$ the component of the vorticity in the $i$ th direction, are displayed in Figures 3(a) and 3(b). These results are compared with filtered DNS quantities at the same filter size, $\bar{\Delta}=16 \Delta x$, with $\Delta x$ the DNS mesh size. The unfiltered DNS quantities are also shown. The analysis of the enstrophy allows to characterize the behavior of the smallest resolved turbulent scales. ${ }^{38,39}$ Figure 3(c) compares the LES kinetic energy spectra with the DNS one. As expected, the gradient model exhibits an unstable behavior. The decay of the kinetic energy is largely under-estimated and a growth of the enstrophy is predicted instead of a decay. This is due to a growth of the energy at the smallest resolved scales, consequence of the gradient model not leading to enough SGS dissipation. The proposed regularization allows to correct this behavior. The DRG and DS models lead to a correct decaying behavior for both kinetic energy and enstrophy. The DS model leads to a slight under-estimation of the enstrophy in comparison with filtered DNS results. This is due to an under-estimation of the energy contained at the smallest resolved scales as shown by Fig. 3(c), because of the over-estimation of the SGS dissipation as found in a priori tests. The DRG model provides a correct prediction of the energy at all the resolved scales.

Figure 4 shows scalar statistics. The resolved scalar variance decay is displayed in Figure 4(a). To characterize the smallest resolved scales behavior, Figure 4(b) shows the evolution of the resolved scalar enstrophy, ${ }^{40} \frac{1}{2}\left\langle\frac{\partial \bar{Z}}{\partial x_{i}} \frac{\partial \bar{Z}}{\partial x_{i}}\right\rangle$. Because the initial scalar field is only composed of large scales, the first stage of the scalar mixing consists in a growth of the scalar enstrophy. This stage corresponds to the generation of smaller mixing scales due to the transport by the velocity field. After this stage, the scalar enstrophy decreases due to the dissipation process. The gradient model over-predicts the growth of the scalar enstrophy and is not able to predict the decay. This implies

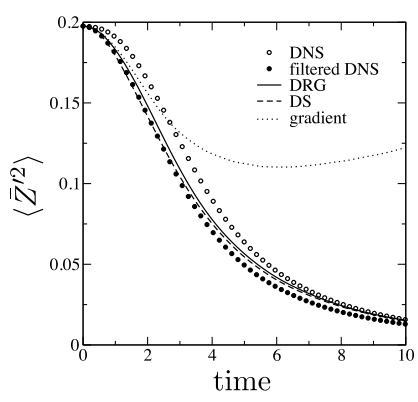

(a)

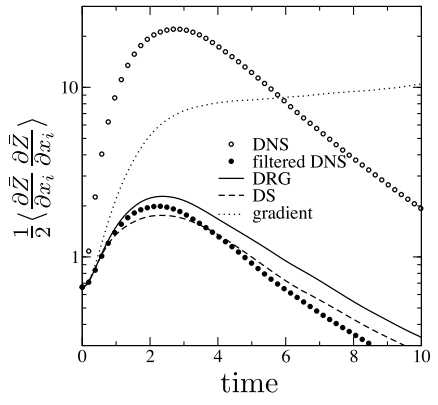

(b)

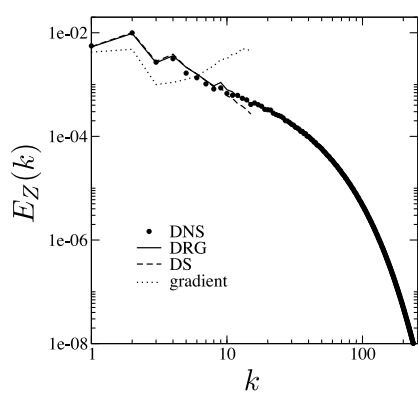

(c)

FIG. 4. Comparison of decaying homogeneous isotropic turbulence statistics: evolution of the resolved scalar variance (a) and scalar enstrophy (b) with time, and scalar variance spectrum at $t=5$ (c). DNS (symbol), DRG model (solid line), DS model (dashed line), and gradient model (dotted line). 
a large under-estimation of the scalar variance decay and even an increase of this quantity. This is due to the incorrect velocity field but also to the gradient model of the SGS scalar flux not predicting enough SGS scalar dissipation. ${ }^{24}$ This eventually leads to a large over-prediction of the scalar variance spectrum at the smallest resolved scales as see in Figure 4(c). As previously observed for the velocity field prediction, the proposed regularization of the gradient model allows to correct this behavior. The decays of both scalar variance and scalar enstrophy are correctly predicted by DS and DRG models, even though a slight over-prediction of the scalar enstrophy is observed. As for the kinetic energy spectrum, the DS model slightly under-predicts the scalar variance spectrum at the smallest resolved scales, whereas the DRG model provides a correct prediction at all the resolved scales. This first a posteriori test confirms the ability of the proposed regularization of the gradient model to correct the well-known unstable behavior of the classic gradient model, which allows to perform stable LES with good performance.

The DRG models set is now tested on other flow configurations including transitional and wall-bounded flows.

\section{B. Turbulent plane jet}

Te mixing of a scalar initially seeded in a turbulent plane jet is computed using the YALES2 flow solver. ${ }^{41}$ This code solves the low-Mach number Navier-Stokes equations for turbulent reactive flows on unstructured meshes using a projection method for constant. ${ }^{42}$ or variable density flows ${ }^{43}$ It relies on fourth-order central finite-volume schemes and on highly efficient linear solvers, ${ }^{44}$ which enable the simulation and the post-processing of iso-thermal, reacting, or multiphase flows on massive unstructured grids. ${ }^{45-47}$ Localized averages in space are used as suggested by Moin et al. ${ }^{12}$ when performing the dynamic procedures of both the SGS stress tensor and the SGS scalar flux models. The flow configuration is similar to the configuration studied by da Silva and Métais. ${ }^{25}$ The inlet boundary condition is given by an analytic hyperbolic tangent profile for both the velocity and scalar fields. A white noise is added to the inlet condition of the velocity field. The Reynolds number is $\operatorname{Re}=\Delta U H / v=3000$, with $H$ the initial thickness of the jet and $\Delta U=U_{j}-U_{c}$ with $U_{j}=1.091$ and $U_{c}=0.091$, respectively, the center jet and co-flow velocity. The scalar field is initially equal to 1 in the jet and to 0 outside. The Schmidt number is fixed to 0.7. The computational domain size is $12.4 \mathrm{H} \times 12 \mathrm{H} \times 2.9 \mathrm{H}$ in the streamwise, normal, and spanwise directions. A DNS is first performed as reference. The DNS mesh is composed of $49.3 \times 10^{6}$ of tetrahedral cells with a roughly uniform size (a edge size is around $0.04 \mathrm{H}$, as in da Silva and Métais ${ }^{25}$ ). The LES is performed on an adapted mesh following a strategy recently proposed. ${ }^{48}$ This mesh is composed of $3.4 \times 10^{6}$ of tetrahedral cells with edge sizes increasing from $0.04 \mathrm{H}$ to $0.3 \mathrm{H}$.

Figure 5 shows the velocity statistics profiles at three distances from the inlet. First, Fig. 5(a) compares the mean streamwise velocity profiles. Both RGM and DS models are in good agreement

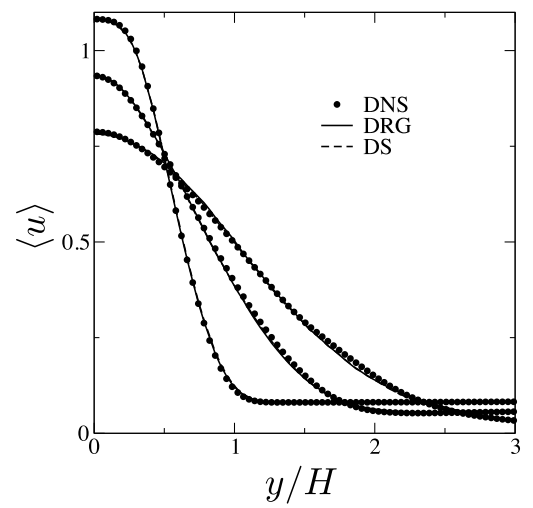

(a)

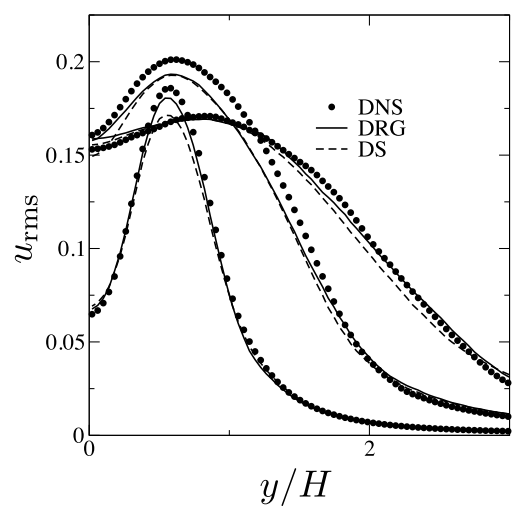

(b)

FIG. 5. Comparison of turbulent plane jet statistics: mean (a) and rms (b) axial velocity profile at three sections, $x / H=4,8$, and 12. DNS (symbol), DRG model (solid line), and DS model (dashed line). 


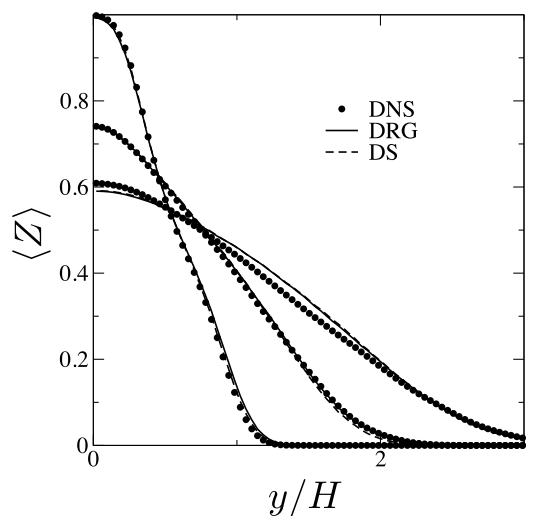

(a)

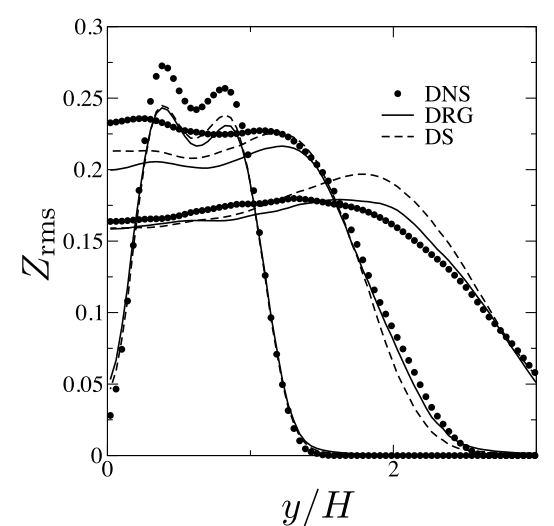

(b)

FIG. 6. Comparison of turbulent plane jet statistics: mean (a) and rms (b) scalar profile at three sections, $x / H=4,8$, and 12 . DNS (symbol), DRG model (solid line), and DS model (dashed line).

with DNS data. Some differences appear on the rms velocity profiles in Fig. 5(b). At $x / H=4$ corresponding to the beginning of the transition, the DRG model better predicts the rms velocity peak, whereas the DS model slightly under-predicts this peak. The rms velocity peak is slightly under-predicted by both models at $x / H=8$. This region corresponds to the end of the potential core and its correct capture by LES remains challenging. Finally, at $x / H=12$ corresponding to the end of the transition, both models have similar prediction in agreement with DNS data. This demonstrates the good capability of the DRG model to deal with flows in transition toward a fully developed turbulence state. Scalar statistics at the same three sections are displayed in Figure 6. Differences with DNS results are more pronounced because they result from disagreement on the velocity field and from the SGS scalar flux model, both appearing in the filtered scalar transport equation. Even though global good agreements are found for both DRG and DS models, the mean scalar profiles exhibit some differences between LES and DNS results at the last section, $x / H=12$. In particular, the scalar value is slightly over-predicted by both models in the shear layer and the scalar value in the center of the jet is slightly under-predicted. Differences are again more visible for rms quantities. During the transition process (at $x / H=4$ and 8), under-estimation persists in the core of the jet. This behavior is more pronounced for the DRG model than for the DS model. This might be due to a complex interaction between modeling errors of velocity and scalar fields. Conversely, at the jet end, the DS model over-predicts the rms value peak in the shear layer. The DRG model allows to slightly correct this behavior even though an over-prediction persists. However,

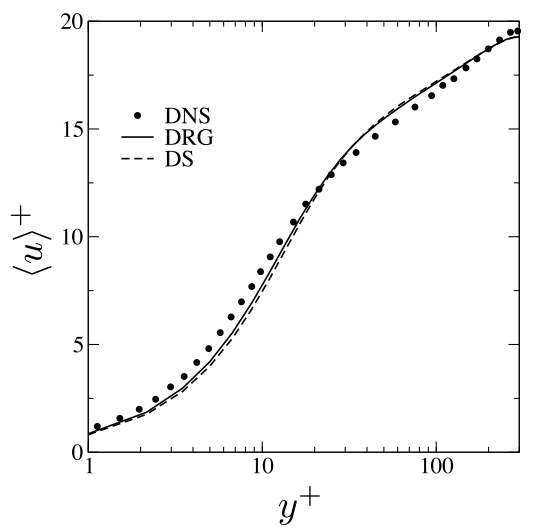

(a)

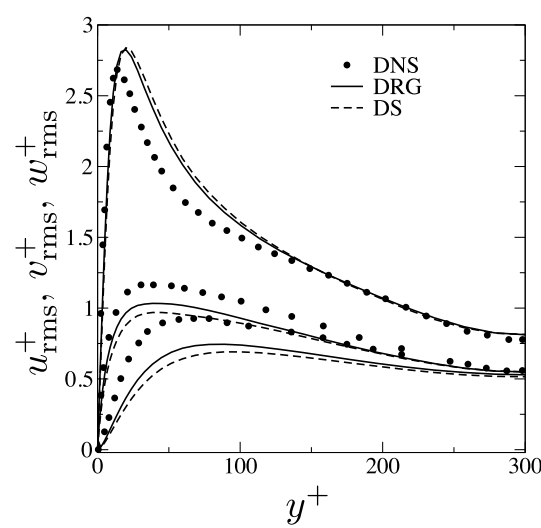

(b)

FIG. 7. Comparison of turbulent channel flow statistics: mean (a) and rms (b) velocity profile. DNS (symbol), DRG model (solid line), and DS model (dashed line). 


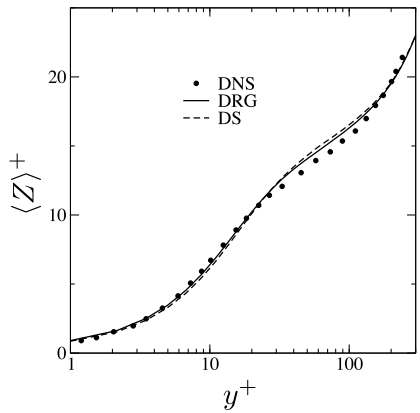

(a)

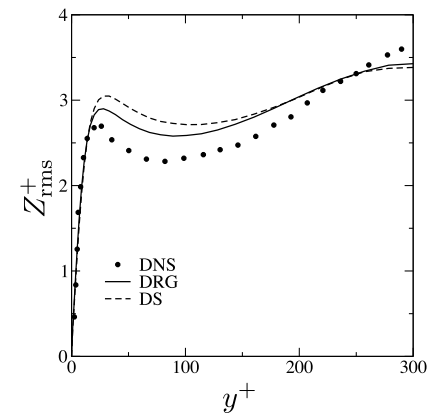

(b)

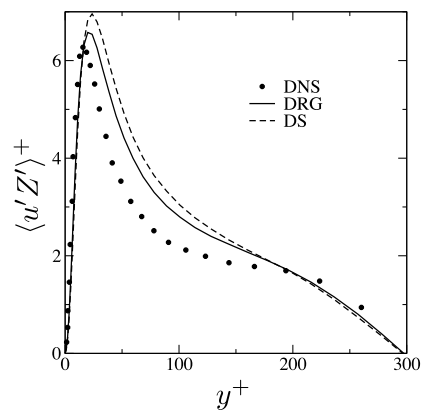

(c)

FIG. 8. Comparison of turbulent channel flow statistics: mean (a) and rms (b) scalar profile, and $\left\langle u^{\prime} Z^{\prime}\right\rangle$ profile (c). DNS (symbol), DRG model (solid line), and DS model (dashed line).

both sets of models have satisfactory behavior, and this test case confirms that the regularization procedure proposed allows stable LES in transitional flow configuration.

\section{Channel flow}

The turbulent flow through a plane channel is eventually considered as a last test case. The Reynolds number based the channel half-height $\delta$ and friction velocity $u_{\tau}=\sqrt{v(\partial\langle u\rangle / \partial y)_{\text {wall }}}$ is 300 . Periodic boundary conditions are imposed in the streamwise and spanwise directions, while no-slip conditions are imposed on the top and bottom walls. By using Dirichlet boundary conditions, the scalar is added to the fluid from the top wall, $Z=1$, and removed from the bottom wall, $Z=-1$. The Schmidt number is fixed to 0.72 . The LES is still performed using YALES2. The computational domain size is $2 \pi \delta \times 2 \delta \times \pi \delta$ in the streamwise, normal, and spanwise direction, and the structured mesh is composed of $48 \times 64 \times 72$ grid points, with a stretching applied in the normal direction to obtain $\Delta y^{+} \approx 0.7$ at the walls.

Figures 7 and 8 display, respectively, the velocity and scalar statistics. The LES results are compared with DNS results extracted from Morinishi et al. ${ }^{49}$ Variables are normalized using wall units. The observations formulated for the previous test case still apply to the present configuration. The mean velocity and scalar profiles predicted by DRG and DS models are similar and in agreement with DNS results. Some differences appear on the fluctuating quantities, and a slight improvement can be noted for the set of DRG models in comparison with the set of DS models. This last test case confirms that the proposed regularization of the gradient model also leads to stable LES in wall-bounded flow, with a correct prediction of both velocity and scalar fields.

\section{CONCLUSION}

In this paper, a novel closure model for the subgrid-scale (SGS) stress tensor appearing in the filtered Navier-Stokes equations is derived in the context of large eddy simulation (LES). The proposed model introduces a regularization of the classic gradient model. The gradient model, which is based on Taylor series expansions of the filtering operation, is known to provide good estimates of the local SGS stress tensor but to yield in the same time unstable behavior when applied to a posteriori tests because of its incorrect prediction of grid scale/subgrid scale (GS/SGS) energy transfers. The proposed regularization first relies on a decomposition of the filtered velocity gradient appearing in the gradient model using the filtered rotation and strain rate tensors. The filtered strain rate tensor can then be split into two tensors, respectively, defined from its positive and negative eigenvalues. The regularized gradient model for the SGS stress tensor is finally defined by keeping only the term leading to direct (forward) GS/SGS energy transfer. This regularization is an extension to the Navier-Stokes equations of a regularization procedure previously proposed for the SGS scalar flux modeling in the context of LES of turbulent mixing. The proposed 
regularization allows to better control the SGS dissipation by avoiding the excessive backscattering predicted by the gradient model. A priori tests demonstrated that the proposed model leads to an improvement of the structural performance in comparison with the classic dynamic Smagorinsky model. In the same time, the proposed regularized gradient model also leads to an improvement of the functional performance in comparison with the gradient model with a better prediction of the global GS/SGS energy transfer. The new set of regularized gradient models for both the filtered momentum and scalar transport equations is finally tested in a posteriori tests by performing LES for various flow configurations. These tests confirm that the regularization allows to stabilize the gradient model. Moreover, in comparison with the classic set of dynamic Smagorinsky models, the new set of models improves the prediction of the flow dynamics and of the turbulent mixing at the smallest resolved scales, as demonstrated by LES of homogeneous isotropic turbulence. Other LES of a turbulent plane jet and of a turbulent channel flow confirm the good behavior of the proposed regularized gradient models.

\section{ACKNOWLEDGMENTS}

This work is supported by the Agence Nationale pour la Recherche (ANR) under Contract No. ANR-2010-JCJC-091601. This work was performed using HPC resources from GENCI-IDRIS (Grant No. 2014-020611) and from CIMENT infrastructure (supported by the Rhône-Alpes region and the Equip@Meso project). Vincent Moureau and Ghislain Lartigue from the CORIA lab, and the SUCCESS scientific group are acknowledged for providing the YALES2 code. LEGI is part of Labex OSUG@2020 (No. ANR10LABX56) and Labex Tec21 (No. ANR11LABX30).

${ }^{1}$ M. Lesieur and O. Métais, "New trends in large-eddy simulations of turbulence," Annu. Rev. Fluid Mech. 28, 45-82 (1996).

${ }^{2}$ C. Meneveau and J. Katz, "Scale-invariance and turbulence models for large-eddy simulation," Annu. Rev. Fluid Mech. 32, 1-32 (2000).

${ }^{3}$ P. Sagaut, Large Eddy Simulation for Incompressible Flows: An Introduction (Springer, 2006).

${ }^{4}$ O. Métais and M. Lesieur, "Spectral large-eddy simulation of isotropic and stably stratified turbulence," J. Fluid Mech. 239, 157-194 (1992).

5 J. Smagorinsky, "General circulation experiments with the primitive equations," Mon. Weather Rev. 91, 99-164 (1963).

${ }^{6}$ A. W. Vreman, "An eddy-viscosity subgrid-scale model for turbulent shear flow: Algebraic theory and applications," Phys. Fluids 16(10), 3670-3681 (2004).

${ }^{7}$ R. S. Rogallo and P. Moin, "Numerical simulation of turbulent flows," Annu. Rev. Fluid Mech. 16, 99-137 (1984)

${ }^{8}$ P. Moin and J. Kim, "Numerical investigation of turbulent channel flow," J. Fluid Mech. 118, 341-377 (1982).

${ }^{9}$ M. Germano, U. Piomelli, P. Moin, and W. H. Cabot, "A dynamic subgrid-scale eddy viscosity model," Phys. Fluids A 3, 1760-1765 (1991).

${ }^{10}$ E. Lévèque, F. Toschi, L. Shao, and J.-P. Bertoglio, "Shear-improved Smagorinsky model for large-eddy simulation of wall-bounded turbulent flows," J. Fluid Mech. 570, 491-502 (2007).

${ }^{11}$ D. K. Lilly, "A proposed modification of the Germano subgrid-scale closure method," Phys. Fluids A 4, 633-635 (1992).

12 P. Moin, K. Squires, W. Cabot, and S. Lee, "A dynamic subgrid-scale model for compressible turbulence and scalar transport,” Phys. Fluids A 3, 2746-2757 (1991).

${ }^{13}$ R. A. Clark, J. H. Ferziger, and W. C. Reynolds, "Evaluation of subgrid-scale models using an accurately simulated turbulent flow,” J. Fluid Mech. 91, 1-16 (1979).

${ }^{14} \mathrm{~K}$. Horiuti, "Roles of non-aligned eigenvectors of strain-rate and subgrid-scale stress tensors in turbulence generation," J. Fluid Mech. 491, 65-100 (2003).

${ }^{15}$ C. Brun, R. Friedrich, and C. B Da Silva, "A non-linear SGS model based on the spatial velocity increment," Theor. Comput. Fluid Dyn. 20(1), 1-21 (2006).

${ }^{16}$ J. Bardina, J. H. Ferziger, and W. C. Reynolds, "Improved subgrid scale models for large eddy simulation,” AIAA Paper No. 80-1357, 1980.

${ }^{17}$ K. W. Bedford and W. K. Yeo, "Conjective filtering procedures in surface water flow and transport," in Large Eddy Simulation of Complex Engineering and Geophysical Flows, edited by B. Galperin and S. A. Orszag (Cambridge University Press, New York, 1993).

18 A. Leonard, "Energy cascade in large-eddy simulations of turbulent fluid flows," Adv. Geophys. A18, 237 (1974).

${ }^{19}$ M. D. Love, "Subgrid modelling studies with Burgers equation," J. Fluid Mech. 100(01), 87-110 (1980).

${ }^{20}$ B. Vreman, B. Geurts, and H. Kuerten, "Large eddy simulation of the temporal mixing layer using the Clark model," Theor. Comput. Fluid Dyn. 8(4), 309-324 (1996).

${ }^{21}$ B. Vreman, B. Geurts, and H. Kuerten, "Large-eddy simulation of the turbulent mixing layer," J. Fluid Mech. 339, 357-390 (1997).

${ }^{22} \mathrm{H}$. Lu and F. Porté-Agel, "A modulated gradient model for scalar transport in large-eddy simulation of the atmospheric boundary layer," Phys. Fluids 25, 015220 (2013).

${ }^{23}$ Y. Fabre and G. Balarac, "Development of a new dynamic procedure for the Clark model of the subgrid-scale scalar flux using the concept of optimal estimator," Phys. Fluids 23, 115103 (2011). 
${ }^{24}$ G. Balarac, J. Le Sommer, X. Meunier, and A. Vollant, "A dynamic regularized gradient model of the subgrid-scale scalar flux for large eddy simulations," Phys. Fluids 25(7), 075107 (2013).

${ }^{25}$ C. B. da Silva and O. Métais, "On the influence of coherent structures upon interscale interactions in turbulent plane jets," J. Fluid Mech. 473, 103-145 (2002).

${ }^{26}$ U. Piomelli, W. H Cabot, P. Moin, and S. Lee, "Subgrid-scale backscatter in turbulent and transitional flows," Phys. Fluids A 3(7), 1766-1771 (1991).

${ }^{27}$ H. S. Kang and C. Meneveau, "Universality of large eddy simulation model parameters across a turbulent wake behind a heated cylinder," J. Turbul. 3, 26 (2002).

${ }^{28}$ B.-C. Wang and D. J. Bergstrom, "A dynamic nonlinear subgrid-scale stress model," Phys. Fluids 17, 035109 (2005).

${ }^{29}$ S. Heinz and H. Gopalan, "Realizable versus non-realizable dynamic subgrid-scale stress models," Phys. Fluids 24, 115105 (2012).

${ }^{30}$ K. Alvelius, "Random forcing of three-dimensional homogeneous turbulence," Phys. Fluids 11, 1880-1889 (1999).

${ }^{31}$ J.-B. Lagaert, G. Balarac, and G.-H. Cottet, "Hybrid spectral-particle method for the turbulent transport of a passive scalar," J. Comput. Phys. 260, 127-142 (2014).

${ }^{32} \mathrm{G}$. Balarac, H. Pitsch, and V. Raman, "Development of a dynamic model for the subfilter scalar variance using the concept of optimal estimators," Phys. Fluids 20(3), 035114 (2008).

${ }^{33}$ G. Balarac, H. Pitsch, and V. Raman, "Modeling of the subfilter scalar dissipation rate using the concept of optimal estimators," Phys. Fluids 20(9), 091701 (2008).

${ }^{34}$ R. Deutsch, Estimation Theory (Prentice-Hall, Englewood Cliffs, NJ, 1965).

${ }^{35}$ A. Moreau, O. Teytaud, and J. P. Bertoglio, "Optimal estimation for large-eddy simulation of turbulence and application to the analysis of subgrid models," Phys. Fluids 18, 1-10 (2006).

36 J. A. Langford and R. D. Moser, "Optimal LES formulations for isotropic turbulence," J. Fluid Mech. 398, 321-346 (1999).

${ }^{37}$ V. Eswaran and S. B. Pope, "Direct numerical simulations of the turbulent mixing of a passive scalar," Phys. Fluids 31, 506-520 (1988).

${ }^{38}$ C. B. da Silva and J. C. F. Pereira, "The effect of subgrid-scale models on the vortices computed from large-eddy simulations," Phys. Fluids 16(12), 4506-4534 (2004).

${ }^{39}$ T. Hughes, L. Mazzei, A. A. Oberai, and A. Wray, "The multiscale formulation of large eddy simulation: Decay of homogeneous isotropic turbulence," Phys. Fluids 13(2), 505-512 (2001).

${ }^{40}$ M. Lesieur, Turbulence in Fluids (Springer, 2008).

${ }^{41}$ V. Moureau, P. Domingo, and L. Vervisch, "Design of a massively parallel CFD code for complex geometries," C. R. Mec. 339, 141-148 (2011)

42 A. J. Chorin, "Numerical solution of the Navier-Stokes equations," Math. Comput. 22(104), 745-762 (1968).

${ }^{43}$ C. D. Pierce and P. Moin, "Progress-variable approach for large-eddy simulation of non-premixed turbulent combustion," J. Fluid Mech. 504, 73-97 (2004).

${ }^{44}$ M. Malandain, N. Maheu, and V. Moureau, "Optimization of the deflated conjugate gradient algorithm for the solving of elliptic equations on massively parallel machines," J. Comput. Phys. 238, 32-47 (2013).

45 V. Moureau, P. Domingo, and L. Vervisch, "From Large-Eddy simulation to direct numerical simulation of a lean premixed swirl flame: Filtered laminar flame-PDF modeling," Combust. Flame 158(7), 1340-1357 (2011).

${ }^{46}$ L. Guedot, G. Lartigue, and V. Moureau, "Design of implicit high-order filters on unstructured grids for the identification of large scale features in les and application to a swirl burner," Phys. Fluids 27, 045107 (2015).

${ }^{47}$ N. Odier, G. Balarac, C. Corre, and V. Moureau, "Numerical study of a flapping liquid sheet sheared by a high-speed stream," Int. J. Multiphase flow 77, 196-208 (2015).

${ }^{48}$ P. Benard, G. Balarac, V. Moureau, C. Dobrzynski, G. Lartigue, and Y. D’Angelo, "Mesh adaptation for large-eddy simulations in complex geometries," Int. J. Numer. Methods Fluids (published online 2015).

${ }^{49}$ Y. Morinishi, S. Tamano, and E. Nakamura, "New scaling of turbulence statistics for incompressible thermal channel flow with different total heat flux gradients," Int. J. Heat Mass Transfer 50(9), 1781-1789 (2007). 